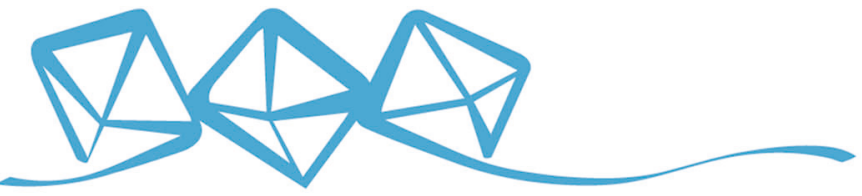 COMMUNICATIONS MATERIALS
}

ARTICLE

https://doi.org/10.1038/s43246-020-0015-4

OPEN

\section{Signatures of complex optical response in Casimir interactions of type I and II Weyl semimetals}

Pablo Rodriguez-Lopez ${ }^{1,2,3}$, Adrian Popescu4 4 , Ignat Fialkovsky5, Nail Khusnutdinov 5,6 \& Lilia M. Woods (1) ${ }^{4 凶}$

The Casimir interaction, induced by electromagnetic fluctuations between objects, is strongly dependent upon the electronic and optical properties of the materials making up the objects. Here we investigate this ubiquitous interaction between semi-infinite spaces of topologically nontrivial Weyl semimetals. A comprehensive examination of all components of the bulk conductivity tensor and the surface conductivity due to the Fermi arc states in real and imaginary frequency domains is presented using the Kubo formalism for materials with different degree of tilting of their linear energy cones. The Casimir energy is calculated using a generalized Lifshitz approach, for which electromagnetic boundary conditions for anisotropic materials were derived and used. We find that the interaction between Weyl semimetals is metallic-like and its magnitude and characteristic distance dependence can be modified by the degree of tilting and chemical potential. The nontrivial topology plays a secondary role in the interaction and thermal fluctuations are expected to have similar effects as in metallic systems.

\footnotetext{
${ }^{1}$ Área de Electromagnetismo, Universidad Rey Juan Carlos, Tulipán s/n, 28933 Móstoles, Madrid, Spain. ${ }^{2}$ Materials Science Factory, Instituto de Ciencia de Materiales de Madrid (ICMM), Consejo Superior de Investigaciones Científicas (CSIC), Sor Juana Inés de la Cruz 3, 28049 Madrid, Spain. ${ }^{3}$ GISC-Grupo Interdisciplinar de Sistemas Complejos, 28040 Madrid, Spain. ${ }^{4}$ Department of Physics, University of South Florida, Tampa, Florida 33620, USA. ${ }^{5}$ Centro de Matemática, Computação e Cognição, Universidade Federal do ABC, 09210-170 Santo André, SP, Brazil. ${ }^{6}$ Regional Scientific and Educational Mathematical

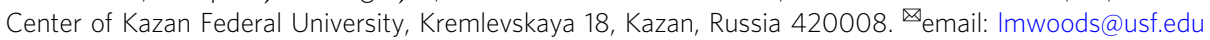


ight-matter interactions are at the forefront of current fundamental and applied research. The exchange of zero-point energy modes of fluctuating electromagnetic fields between objects with finite boundaries gives rise to the ubiquitous Casimir interaction $^{1}$. This type of force is strongly dependent on the geometry of the objects as well as their electronic and optical response $^{2}$. The Casimir force is especially pronounced at micro and submicrometer separations and it can limit the operation of nano and micro electronic devices due to unwanted sticktion and adhesion $^{3}$. Recent developments have shown that it is beneficial to explore the materials aspect of Casimir interactions in order to probe novel physics ${ }^{4}$. This in turn can be of great importance to find ways to control the magnitude and sign of this ubiquitous force, which may be used to improve the performance of tiny devices.

Materials with Dirac spectrum have been a fruitful platform for Casimir force discoveries. Perhaps the most studied system in this regard has been graphene whose reduced dimensionality and linear energy band structure led to many unusual functionalities in this interaction, including much enhanced thermal effects $^{5-12}$. Additionally, Casimir force phase transitions driven by external fields have been predicted in related $2 \mathrm{D}$ materials, such as silicene, germanene, and stanene ${ }^{13}$. Moreover, repulsive and force quantization effects have been described in other materials, such as 3D topological insulators and Chern insulators $^{14-17}$. Weyl semimetals (WSMs) are $3 \mathrm{D}$ materials and they have analogous to graphene linear energy dispersion band structure near the Fermi level. WSMs are also characterized by linear energy cones, whose nodes can be viewed as magnetic monopoles in reciprocal space, associated with Berry curvature $\mathrm{e}^{18-20}$. Tilting of the Weyl cones is also possible as one distinguishes between type I and type II WSMs depending on the degree of tilting ${ }^{21-23}$. Exotic surface states, which are associated with the nontrivial topology in these materials have also been demonstrated 19,20 .

All of these unique electronic structure properties have a direct consequence in the optical response of Weyl semimetals. The interplay between the 3D linear energy dispersion, separations of the Weyl cones, and their tilting results in an anisotropic optical conductivity tensor with distinct features in the longitudinal and Hall components, as well as the existence of surface conductivity due to the special surface Fermi arc states. In this work, using an effective model for the linear band structure and the Kubo formalism a comprehensive examination of the different optical conductivity components is presented for type I and II WSMs. The Casimir interaction is calculated using a generalized Lifshitz approach for which the boundary conditions for anisotropic media are resolved via the Fresnel reflection matrix. We focus on the purely quantum mechanical and purely thermal regimes, which helps understand how different features in the optical conductivity affect the interaction. Although the optical response is complex, the Casimir interaction is found to be dominated by the bulk diagonal conductivity components, while the surface states and Hall conductivity play a secondary role. Unlike 2D Dirac materials for which several unusual functionalities were found due to their non-trivial topology, the Casimir interaction in WSMs is similar to the one for metallic systems. We find that the magnitude of the interaction is strongly affected by the cutoff energy for the linear band dispersion, the chemical potential, and the cone tilting.

\section{Results and discussion}

Electronic properties of Weyl semimetals. The essential electronic properties of Weyl materials with linearly dispersing bands crossing at the Fermi level can be captured by the following
Hamiltonian per Weyl node 19,24

$$
H_{\eta}=\left(\eta \hbar b_{0}+\mathbf{v}_{\mathbf{t}} \cdot \mathbf{k}\right) \mathbf{1}+\hbar v_{\mathrm{F}} \boldsymbol{\sigma} \cdot(\eta \mathbf{k}+\mathbf{b}),
$$

with eigenenergies

$$
E_{\mathbf{k}}^{\eta}=\left(\eta \hbar b_{0}+\mathbf{v}_{\mathrm{t}} \cdot \mathbf{k}\right) \pm \hbar v_{\mathrm{F}}|\eta \mathbf{k}+\mathbf{b}| .
$$

This is a minimal model with a pair of Weyl nodes specified by their chirality $\eta= \pm 1$. Here $v_{\mathrm{F}}$ is the Fermi velocity, $\boldsymbol{\sigma}=\left(\sigma_{\mathrm{x}}, \sigma_{\mathrm{y}}, \sigma_{\mathrm{z}}\right)$ are the Pauli matrices, $\mathbf{k}$ is the $3 \mathrm{D}$ wave vector, and $\mathbf{1}$ is the $2 \times 2$ identity matrix. The nontrivial topology corresponding to the above Hamiltonian is determined by the separation between the two crossing points in the band structure. For systems with broken Inversion symmetry this separation, denoted as $b_{0}$, marks the distance between the Weyl points in energy space, while for systems with broken time reversal symmetry $\mathbf{b}$ gives this separation in momentum space. These two cases are generalized in Eq. (1) by using the four-vector $b_{\mu}=\left(b_{0}, \mathbf{b}\right)$.

In addition to their separation, the Weyl cones can also be tilted, which is determined by the tilt velocity $\mathbf{v}_{\mathrm{t}}$. One distinguishes between $v_{\mathrm{t}} / v_{\mathrm{F}}<1$ and $v_{\mathrm{t}} / v_{\mathrm{F}}>1$ cases corresponding to type I and type II Weyl semimetals, respectively. The $\mathbf{v}_{t}=$ 0 situation corresponds to the simplest type I material with cones in an upright position (no tilting). In type II WSM the tilt is large enough so the cones are tipped over transforming the point-like Fermi surface, typical for type I WSM, to a different shape. Due to the $v_{\mathrm{t}} / v_{\mathrm{F}}>1$ condition, there is also a finite density of states at the Fermi level characterized by electron and hole pockets. Let us note that the nontrivial topology is irrelevant of the cone tilting and it is present in both types of semimetals. Nevertheless, many properties are expected to be affected by $v_{\mathrm{t}}$ and its relative value with respect to $v_{\mathrm{F}}$, giving rise to distinct features in the optical response, anisotropic chiral anomaly, and anomalous Hall effects among others ${ }^{24-28}$.

In this work we consider the separation between the cones as described by $b_{\mu}=\left(b_{0}, b \hat{\boldsymbol{k}}_{\mathrm{z}}\right)$ and we choose the cone tilting with respect to the $z$-axis in momentum space with $\mathbf{v}_{\mathrm{t}}=v_{\mathrm{t}} \hat{\boldsymbol{k}}_{\mathrm{z}}$. As a result, the above Hamiltonian and its eigenergies and eigenspinors can be simplified to

$$
\begin{gathered}
H_{\eta}=\left(\hbar b_{0}+\Psi K_{\mathrm{z}}^{\eta}\right) \mathbf{1}+\boldsymbol{\sigma} \cdot \boldsymbol{K}^{\eta}, \\
E_{\mathbf{k}}^{\eta, \pm}=\left(\eta \hbar b_{0}+\Psi \hbar v_{\mathrm{F}} k_{\mathrm{z}}\right) \pm\left|\boldsymbol{K}^{\eta}\right|, \\
\left|u_{\boldsymbol{k}}^{\eta}\right\rangle=\frac{1}{\sqrt{2\left|\boldsymbol{K}^{\eta}\right|\left|\boldsymbol{M}^{\eta}\right|}}\left(\begin{array}{c}
\frac{\left|\boldsymbol{M}^{\eta}\right|\left(K_{\mathrm{x}}^{\eta}+\mathrm{i} K_{\mathrm{y}}^{\eta}\right)}{K_{\perp}^{\eta}} \\
\hbar v_{\mathrm{F}} K_{\perp}^{\eta}
\end{array}\right),
\end{gathered}
$$

where $\boldsymbol{K}^{\eta}=\hbar v_{\mathrm{F}}\left(\eta \boldsymbol{k}+b \hat{\boldsymbol{k}}_{\mathrm{z}}\right), \quad K_{\perp}^{\eta}=\sqrt{K_{\mathrm{x}}^{\eta, 2}+K_{\mathrm{y}}^{\eta, 2}}, \quad$ and $\left|\boldsymbol{M}^{\eta}\right|=$ $\left|\boldsymbol{K}^{\eta}\right|+K_{\mathrm{z}}^{\eta}$. Also, $\Psi=v_{\mathrm{t}} / v_{\mathrm{F}}$ denotes the tilting ratio. In Fig. 1a, we show representative band structures for type I WSM whose cones are separated in momentum space, which corresponds to materials with broken Time Reversal symmetry. Fig. 1b shows type II WSM with cones separated along the energy direction, which reflects systems with broken Inversion symmetry. Due to the tilted cones in type II WSM free carriers at the Fermi level are also available as schematically shown in Fig. 1c.

Optical response of Weyl semimetal. Using the eigenstates and energies from Eqs. $(4,5)$ the conductivity tensor for both types of WSMs is obtained using the Kubo formalism and it is found of 

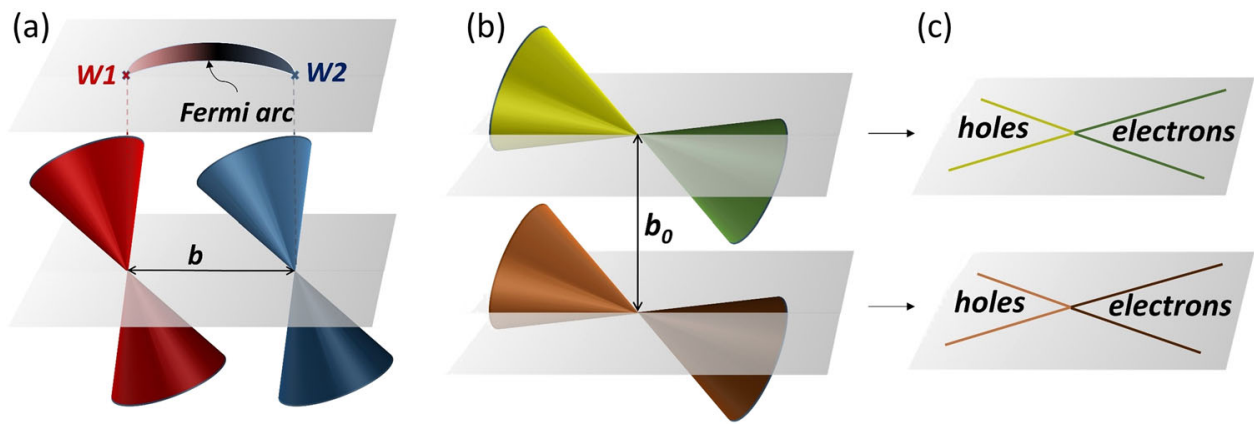

Fig. 1 Graphical representation of the electronic structure of Weyl semimetals. a Separated cones in momentum space by $b$ with tilting $\Psi<1$. The projected bulk Weyl nodes on the surface of the crystal lead to Fermi arc surface states; $\mathbf{b}$ Separated cones in energy space by $b_{0}$ with tilting $\Psi>1$; $\mathbf{c}$ The severe tilting of the cones results in electron and hole states residing on the Fermi surface.

the following form

$$
\boldsymbol{\sigma}^{\eta}=\left[\begin{array}{ccc}
\sigma_{\mathrm{xx}, \mathrm{b}}^{\eta} & \sigma_{\mathrm{xy}, \mathrm{b}}^{\eta} & 0 \\
-\sigma_{\mathrm{xy}, \mathrm{b}}^{\eta *} & \sigma_{\mathrm{xx}, \mathrm{b}}^{\eta} & 0 \\
0 & 0 & \sigma_{\mathrm{zz}, \mathrm{b}}^{\eta}
\end{array}\right] .
$$

Each bulk component is decomposed into interband and intraband terms, such that $\sigma_{\mathrm{ij}, \mathrm{b}}^{\eta}=\sigma_{\mathrm{ij}, \mathrm{b}, \mathrm{i}, \eta}^{\mathrm{int}}+\sigma_{\mathrm{ij}, \mathrm{b}, \mathrm{i}, \eta}$. To obtain the total conductivity corresponding to the Hamiltonian in Eq. (3) summation of the contributions from the Weyl cones must be done, such that $\sigma=\sigma^{(+)}+\sigma^{(-)}$. Detailed calculations and explicit expressions for zero temperature, $T=0$, and no spatial dispersion, $\mathbf{q}=0$, for the Real and Imaginary parts of the interband and intraband conductivity components are given in the Supplementary Note 1.

Our results indicate that both types of materials have anisotropic optical properties with distinct diagonal components. Eqs. (3-5) correspond to the $3 \mathrm{D}$ bulk band structure, which is responsible for $\sigma_{\mathrm{zz}, \mathrm{b}} \neq \sigma_{\mathrm{xx}, \mathrm{b}}=\sigma_{\mathrm{yy}, \mathrm{b}}$. In addition, the nontrivial topology of the separated Weyl cones results in a surface conductivity (discussed later), which gives rise to an anisotropic surface conductivity tensor. The bulk components have an explicit dependence upon the cone tilting and the cutoff energy $\epsilon_{\mathrm{c}}$, as found by several other authors ${ }^{29-33}$. The cutoff energy serves as a limit beyond, which the Dirac bands are not of linear dispersion anymore ${ }^{34,35}$. This is different than the situation in graphene materials, where the optical response is found to be independent of such a cutoff ${ }^{36}$. The unique role of $\epsilon_{\mathrm{c}}$ is thus related to the interplay between the linear band dispersion and dimensionality of the material.

The general expressions for the different conductivity components are quite complicated (see Supplementary Note 1). Thus in Fig. 2 we show the nonzero components for a single cone for both types of WSMs and their dependence on frequency. For $\omega \rightarrow 0, \operatorname{Re}[\sigma]_{\mathrm{xx}, \mathrm{b}}$ and $\operatorname{Re}[\sigma]_{z z, \mathrm{~b}}$ of type I WSM tend to zero when $\mu=0$ (Fig. 2a), while these components exhibit Drude-like behavior for type II WSM due to the finite carrier concentration originating from the severe tilting (Fig. 2c). The Drude-like behavior is also seen in Fig. 2e, g, in which intraband contributions from the non-zero chemical potential are included. We find that $\operatorname{Re}[\sigma]_{\mathrm{xx}, \mathrm{b}}$ and $\operatorname{Re}[\sigma]_{\mathrm{zz}, \mathrm{b}}$ exhibit a linear dependence with frequency until $2 \epsilon_{c}$, however there is some nonlinearity in the $\left(\Omega_{\mathrm{L}}=\frac{2 \mu}{|\Psi-1|}, \Omega_{\mathrm{U}}=\frac{2 \mu}{|\Psi+1|}\right)$ region in Fig. $2 \mathrm{e}, \mathrm{g}^{30}$. The real part of the Hall conductivity shows a discontinuous behavior as $\hbar \omega$ approaches $\epsilon_{\mathrm{c}}$. The imaginary parts of the different components are also given as a function of frequency. The $\operatorname{Im}[\sigma]_{\mathrm{xx}, \mathrm{b}}$ and
$\operatorname{Im}[\sigma]_{z z . \mathrm{b}}$ are discontinuous as $\hbar \omega$ approaches $\epsilon_{\mathrm{c}}$ (Fig. $2 \mathrm{~b}, \mathrm{~d}, \mathrm{f}, \mathrm{h}$ ). At small frequency the imaginary parts are mainly determined by the Drude-like behavior $29,31,32$.

In addition to the graphical representation of the WSMs optical response in Fig. 2, analytical expressions in different limits for the plasma frequencies and Hall response can be found. Specifically, in both cases the intraband conductivities are of Drude type with plasma frequencies $\omega_{\mathrm{P}}^{\mathrm{I} \text { II }}$ explicitly dependent on the WSM type, as shown in Supplementary Note 1. In addition, the plasma frequencies for WSM type II are found to diverge as a function of the cutoff, consistent with other works ${ }^{29,32}$. For example, in the limit of large $\epsilon_{\mathrm{c}}$ the xx-component is obtained as

$$
\omega_{\mathrm{P}, \mathrm{xx}}^{\mathrm{II}} \approx \frac{\alpha c}{8 \pi^{2} \hbar v_{\mathrm{F}}}\left[\left(\frac{\Psi^{2}-1}{\Psi}\right) \epsilon_{\mathrm{c}}^{2}-2 \frac{\tilde{\mu}^{2}}{\Psi^{3}} \log \left(\epsilon_{\mathrm{c}}\right)\right],
$$

where $\tilde{\mu}=\left|\mu-\hbar b_{0}\right|$. Similar second power law and logarithimic divergencies upon $\epsilon_{\mathrm{c}}$ are present for the zz-component of the bulk intraband conductivity.

It is also interesting to examine the behavior of the plasma frequencies as a function of chemical potential. For type I WSM $\lim _{\tilde{\mu} \rightarrow 0} \omega_{\mathrm{P}, \mathrm{xx}(\mathrm{zz})}^{\mathrm{I}}=0$, which shows vanishing intraband conductivity as the chemical potential passes through the Fermi level. For type II WSM, one finds that

$$
\lim _{\tilde{\mu} \rightarrow 0} \omega_{\mathrm{P}, \mathrm{xx}}^{\mathrm{II}}=\frac{\alpha c}{8 \pi^{2} \hbar v_{\mathrm{F}}} \frac{\Psi^{2}-1}{\Psi}\left(\epsilon_{c}^{2}+\left(\hbar v_{\mathrm{F}} b\right)^{2}\right) .
$$

Thus even if $\tilde{\mu}=0$, there is a nonzero intraband conductivity. This correlates with the severe tilting in the WSM from $\Psi>1$ which results in a band crossing at the Fermi level, meaning that there are always free carriers available in the system (Fig. 1c).

We further study the Hall conductivity for each type of WSM. The zero frequency limit, which is a measure of the anomalous Hall effect, is obtained as

$$
\begin{aligned}
& \sigma_{\mathrm{xy}}^{\mathrm{I}, \eta}(\omega=0)=\frac{\alpha c}{4 \pi^{2}}\left[\hbar b+\frac{\eta \tilde{\mu}}{\hbar v_{\mathrm{F}}} \frac{\tanh ^{-1}(\Psi)-\Psi}{\Psi^{2}}\right], \\
& \sigma_{\mathrm{xy}}^{\mathrm{II}, \eta}(\omega=0)=\frac{\alpha c}{4 \pi^{2}}\left[\hbar b+\frac{\eta \tilde{\mu}}{\hbar v_{\mathrm{F}} \Psi^{2}} \log \frac{\epsilon_{\mathrm{c}} \Psi \sqrt{\Psi^{2}-1}}{\tilde{\mu}}\right] .
\end{aligned}
$$

The first term in the above expressions is common to both types of WSMs. It is proportional to the distance $b$ between the cones in momentum space and it reflects the nontrivial topology of the materials. The second term in each $\sigma_{\mathrm{xy}}^{\mathrm{I}(\mathrm{II}), \eta}$ comes from the tilting of the cones and the chemical potential scaled by the separation in energy space $\tilde{\mu}=\left|\mu-\hbar b_{0}\right|$ and it does not have topological origin. The Hall conductivity of type II WSM also shows a 

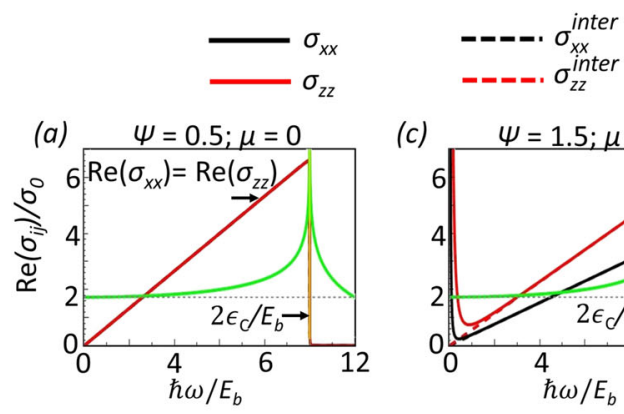

(c)
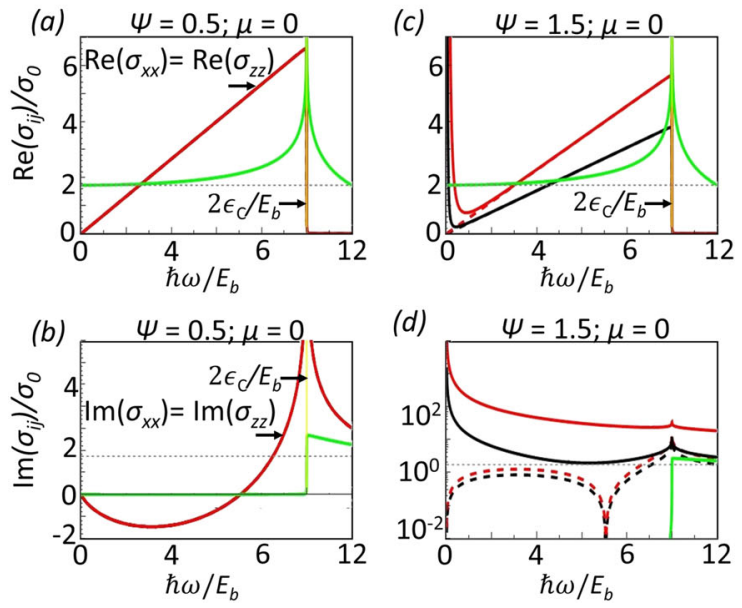

(d)

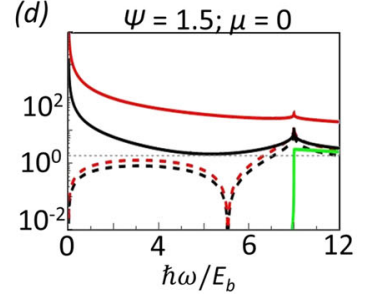

$\sigma_{x y}$ $\ldots \ldots \ldots \sigma_{T}=\alpha c b / 4 \pi^{2}$

(e)

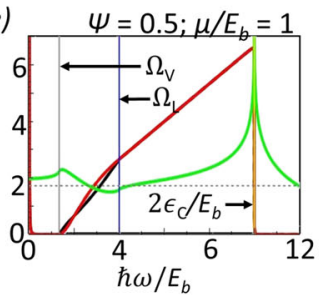

(f)

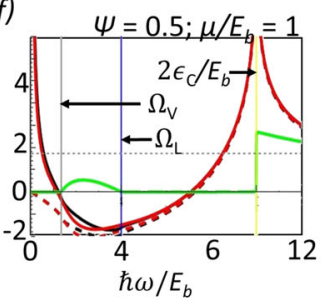

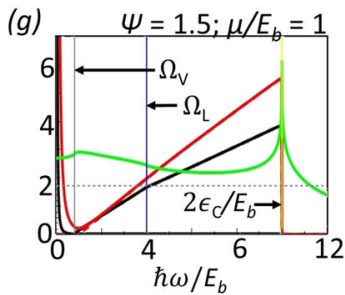

(h)

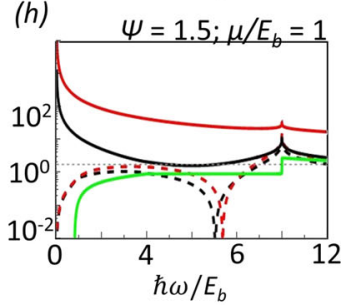

Fig. 2 Conductivity tensor components for type I and II Weyl semimetals at zero temperature and real frequencies. Real and Imaginary parts of different conductivity tensor components for a single Weyl cone scaled by $\sigma_{0}=\frac{\alpha c}{16 \pi v_{\mathrm{F}}}$ as a function of frequency scaled by $E_{\mathrm{b}}=\hbar v_{\mathrm{F}} b$ for: $\mathbf{a}, \mathbf{b} \Psi=0.5, \mu=0 ; \mathbf{c}, \mathbf{d} \Psi=$ $1.5, \mu=0 ; \mathbf{e}, \mathbf{f} \Psi=0.5, \mu / E_{\mathrm{b}}=1 ; \mathbf{g}, \mathbf{h} \Psi=1.5, \mu / E_{\mathrm{b}}=1$. The legend given above the panels denotes the total $\sigma_{\mathrm{xx}, \mathrm{b}}$ (full black) and $\sigma_{\mathrm{zz}, \mathrm{b}}$ (full red), interband contributions $\sigma_{\mathrm{xx}, \mathrm{b}}^{\text {inter }}$ (dashed black) and $\sigma_{\mathrm{xx}, \mathrm{b}}^{\text {inter }}$ (dashed red), the bulk Hall conductivity $\sigma_{\mathrm{xy}, \mathrm{b}}$ (full green) and the topological Hall conductivity $\sigma_{\mathrm{T}}$ (dashed gray). The following parameters were used in the calculations: $\epsilon_{\mathrm{c}}=10 \mathrm{eV}, E_{\mathrm{b}}=1 \mathrm{eV}, \mathrm{c} / \mathrm{v}_{\mathrm{F}}=300, \Gamma / E_{\mathrm{b}}=10^{-3}$.

logarithmic divergence with the energy cutoff, while $\sigma_{\mathrm{xy}}^{\mathrm{I}, \eta}$ is finite at the large $\epsilon_{\mathrm{c}}$ limit. Our results are consistent with calculations reported by other authors ${ }^{31,33}$. Let us note, however that the total Hall conductivity for both types of Weyl semimetals is $\sigma_{\mathrm{xy}}^{\mathrm{I}(\mathrm{II})}(\omega=$ $0)=\frac{\alpha c h b}{4 \pi^{2}}$ as the second terms in Eqs. $(8,9)$ cancel out upon addition due to the opposite in sign cone chiralities.

Surface conductivity due to Fermi arcs. One of the hallmarks of WSMs is their Fermi arc surface states. Other materials support Fermi surface states that have circular or deformed circular shapes $^{37,38}$. In a Weyl semimetal occupying semi-infinite space with an abrupt boundary, however, only half of this usual shape resides on the surface giving rise to a Fermi arc, which essentially starts and ends at the surface projections of the bulk Weyl points, as schematically shown in Fig. 1a. The Fermi arcs are also a signature of the nontrivial bulk topology of WSMs regardless of tilting and their existence is determined by the bulk-surface correspondence ${ }^{39-43}$. In the case of Weyl cones separated in momentum space the Fermi arc can be represented as a single Dirac fermion (similar to graphene), whose conductivity in the low frequency is of Drude-like type and it is determined by the distance between the cones ${ }^{44}$. Specifically, for the case of $b_{\mu}=$ $\left(0, b \hat{\mathbf{k}}_{\mathrm{z}}\right)$ at the $x=0$ surface one finds there is only one nonzero conductivity component

$$
\sigma_{\mathrm{yy}, \mathrm{s}}=\frac{-i \alpha c v_{\mathrm{F}} b}{2 \pi^{2}(\omega+i \Gamma)} .
$$

At the $y=0$ boundary, the nonzero surface conductivity is $\sigma_{\mathrm{xx}, \mathrm{s}}$ with the same value as the above equation. There is no surface conductivity at the $z=0$ boundary since the projection of the vector $b_{\mu}=\left(0, b \hat{\boldsymbol{k}}_{\mathrm{z}}\right)$ is zero in this case $\mathrm{e}^{44}$. Thus this optical response is associated with the WSM boundary due to the distance between the cones $b$ and it is highly anisotropic.

Optical response at imaginary frequency. For the Casimir interaction the conductivity components in imaginary frequency domain $\omega=i \xi$ are needed. Thus it is convenient to use the
Kramers-Krönig relations for which,

$$
\begin{aligned}
\operatorname{Re}\left[\sigma_{\mathrm{ij}}(\mathrm{i} \xi)\right] & =\frac{2}{\pi} \int_{0}^{\infty} d \omega \frac{\xi}{\omega^{2}+\xi^{2}} \operatorname{Re}\left[\sigma_{\mathrm{ij}}(\omega)\right] \\
& =\frac{2}{\pi} \int_{0}^{\infty} d \omega \frac{\omega}{\omega^{2}+\xi^{2}} \operatorname{Im}\left[\sigma_{\mathrm{ij}}(\omega)\right] .
\end{aligned}
$$

Realizing that $\operatorname{Im}\left[\sigma_{\mathrm{ij}}(\mathrm{i} \xi)\right]=0$, the analytical continuation of $\operatorname{Re}\left[\sigma_{\mathrm{ij}}(\mathrm{i} \xi)\right]$ to all positive imaginary frequencies in the upper half of the complex plane gives the final expressions for the conductivity components. By taking $\xi \rightarrow \xi+\hbar / \tau$ finite dissipation can be included in the optical response $(\Gamma=\hbar / \tau)$.

In Fig. 3 results for some functional dependences of the conductivity components (shown explicitly in Supplementary Note 2), commensurate with the ones in Fig. 2, for a single cone at imaginary frequency $\omega=i \xi$ are given. In all cases $\sigma_{\mathrm{xx}, \mathrm{b}}^{\text {inter }}$ and $\sigma_{\mathrm{zz}, \mathrm{b}}^{\text {inter }}$ increase linearly in the small $\xi$ region (Fig. $3 \mathrm{a}-\mathrm{d}$ ). The Hall conductivity has a very weak dependence upon $\xi$ and in both WSMs the $\xi=0$ limit is governed by the analytical expressions in Eqs. $(6,7)$. There is a discontinuous jump at $\Psi=1$, which marks the transition between type I and type II WSMs as shown in Fig. 3e. As $\Psi$ increases for type II WSM the conductivity is determined primarily by the intraband contributions due to increasing the number of free carriers from the severe tilting even if $\mu=0$. Also, $\sigma_{\mathrm{ij}, \mathrm{b}}$ as a function of $\mu$ is depicted for type I WSM in Fig. $3 \mathrm{e}$ at $\xi=0$. This behavior is consistent with our analytical considerations and it shows that the response is mainly determined by the intraband contributions as the chemical potential grows.

Many of the topologically nontrivial features in Weyl semimetals are related to the separation of their energy cones in momentum space. In order to better understand this issue in the context of the Casimir interaction, we consider some limiting cases with respect to $b_{\mu}=\left(0, b \hat{\boldsymbol{k}}_{\mathrm{z}}\right)$. The so obtained results are then compared with the numerical calculations, in which the full optical response of the materials is taken into account.

Casimir interaction for coinciding Weyl cones. At this point, we consider non-tilted Weyl cones with $b=0$. Such a situation with 

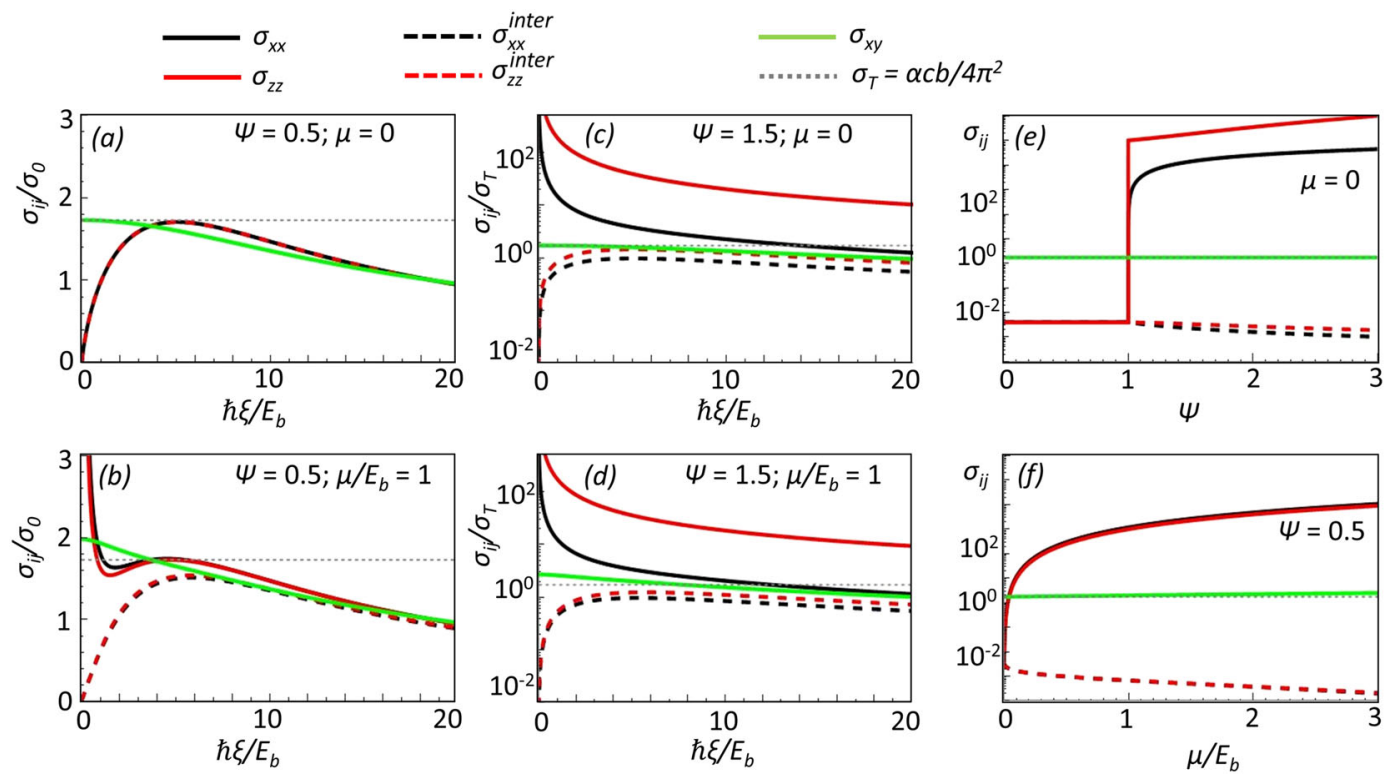

Fig. 3 Conductivity tensor components for type I and II Weyl semimetals at zero temperature and imaginary frequencies. Different contributions to the bulk conductivity components $\sigma_{\mathrm{ij}}$ scaled by $\sigma_{0}=\frac{\alpha \hbar c}{16 \pi h v_{\mathrm{F}}}$ as a function of imaginary frequency $\xi$ scaled by $E_{b}=\hbar v_{\mathrm{F}} b$ for: a $\Psi=0.5, \mu=0 ; \mathbf{b} \Psi=0.5, \mu / E_{\mathrm{b}}=1$; c $\Psi=1.5, \mu=0 ; \mathbf{d} \Psi=1.5, \mu / E_{\mathrm{b}}=1$. Different contributions to the bulk conductivity components $\sigma_{\mathrm{ij}, \mathrm{b}}$ scaled by $\sigma_{0}$ at $\xi=0$ as a function of: e $\Psi$ for $\mu=0$ and $\mathbf{f} \mu / E_{\mathrm{b}}$ for $\Psi=0.5$. The following parameters were used in the calculations: $\epsilon_{\mathrm{c}}=10 \mathrm{eV}, E_{\mathrm{b}}=1 \mathrm{eV}, \mathrm{c} / v_{\mathrm{F}}=300, \Gamma / E_{\mathrm{b}}=10^{-3}$.

coinciding cones corresponds to a 3D Dirac semimetal ${ }^{21}$. In this case, one finds that the interband conductivity components in the imaginary frequency domain are of the following form

$$
\sigma_{\mathrm{ij}}^{\mathrm{inter}}(\mathrm{i} \xi)=\frac{\alpha c \xi}{12 \pi^{2} v_{\mathrm{F}}} \log \left(\frac{\hbar^{2} \xi^{2}+4 \epsilon_{\mathrm{c}}^{2}}{\hbar^{2} \xi^{2}+4 \mu^{2}}\right) \delta_{\mathrm{ij}} .
$$

This isotropic response simplifies the reflection matrices entering in Eq. (20) and some asymptotic behavior of the Casimir energy can be found analytically. For example, the quantum mechanical $(T=0)$ Casimir energy in the limit of large cutoff frequency is obtained as the energy for perfect metals, such that $\frac{E_{\mathrm{M}}}{A}=-\frac{\pi^{2} \hbar c}{720 d^{3}}$. Since $\epsilon_{\mathrm{c}}$ is finite, the limiting cases of large and small separations with respect to the characteristic distance $d_{0}=\frac{\hbar c}{2 \epsilon_{\mathrm{c}}}$ can be found

$$
\begin{gathered}
\lim _{d \ll d_{0}} E=\frac{45}{\pi^{4}} \frac{d}{d_{0}} E_{\mathrm{M}} \times\left[\frac{1}{12 \beta^{2}}+1+\beta+e^{\beta}(\beta+2) \beta \operatorname{Ei}(-\beta)\right], \\
\lim _{d \gg d_{0}} E=\frac{45}{\pi^{4}} \frac{X^{3}-3 X+2}{X(1+X)^{2}} E_{\mathrm{M}},
\end{gathered}
$$

where $\beta=\frac{3 \pi v_{\mathrm{F}}}{\alpha c}, X=\sqrt{1+\frac{1}{\beta} \log \left(1+\frac{\mathrm{d}^{2}}{\mathrm{~d}_{0}^{2}}\right)}$, and $\operatorname{Ei}(x)$ is the exponential integral evaluated at $x$. Thus the small $d$ limit results in a $d^{-2}$ scaling law for the energy. Due to the weak logarithmic dependence on $d$ in $X$, however, the large $d$ limit is essentially similar to the one for perfect metals with a $d^{-3}$ scaling law in the energy.

In Fig. 4a we show the numerical calculations for the quantum mechanical interacting energy of materials with optical response from Eq. (12). These results indicate that the energy cutoff practically determines the characteristic behavior of the Casimir energy through the distance $d_{0}$, which is in agreement with Eqs. $(13,14)$. Specifically, for $\epsilon_{\mathrm{c}}=1 \mathrm{eV}$ one finds that $d_{0}=$ $100 \mathrm{~nm}$, while for $\epsilon_{\mathrm{c}}=5 \mathrm{eV}, d_{0}=20 \mathrm{~nm}$. Since the quantum mechanical Casimir interaction is domated by Matsubara frequencies with $\hbar \xi<\epsilon_{\mathrm{c}}$, the onset of a metallic-like interaction is strongly related to the range of validity of the linear band structure approximation controlled by $\epsilon_{\mathrm{c}}$. For separations smaller than $d_{0}$ one needs to include bands beyond the linear energy approximation studied here. Such an explicit dependence upon the cutoff energy is not present in 2D materials with Dirac energy spectra, where the optical response and Casimir energy are independent of the bandwidth of the linear bands, except at very small distances ${ }^{13,15,36}$.

The role of cone separation in the interaction. The parameter $b$ enters into the reflection matrices in a complicated way, which makes it difficult to obtain analytical expressions for the Casimir energy for the considered materials. To get some idea about the role of the cone separation, we consider the situation when there is no surface conductivity and the bulk conductivity tensor contains only off-diagonal components with $\sigma_{\mathrm{xy}, \mathrm{b}}^{\eta}=\sigma_{\mathrm{T}}=\frac{e^{2} b}{4 \pi^{2}}$, which capture the anomalous Hall effect Eqs. $(8,9)$. We find that in the limiting case of small distance, the Casimir energy is

$$
\lim _{d \rightarrow 0} \frac{E}{A}=\frac{\hbar c \alpha^{2}}{96 \pi^{4} d} b_{1} b_{2}
$$

where $b_{1,2}$ are the cone separation parameters for the two interacting materials. In the large distance limit, the energy for perfect metals is obtained.

Equation 15 shows a $d^{-1}$ scaling law in the short distance separation and the $\alpha^{2}$ dependence in the numerator indicates that the energy is of rather small magnitude. It is also recognized that the product $b_{1} b_{2}$ can be positive or negative given that the cone separation can be in positive or negative domains in momentum space $^{21,22}$. This behavior is reminiscent of the Casimir interaction in other topological materials, whose response is dominated by the Hall conductivity ${ }^{13-15}$. The positive sign of the product of the cone separation parameters, which are essentially proportional to the constant anomalous Hall conductivity, is associated with repulsion as found in $3 \mathrm{D}$ topological insulators and $2 \mathrm{D}$ Chern insulators. However, since $b_{1,2}$ are continuous, the Casimir energy is not quantized as found in other topological materials due to the discrete nature of their Hall conductivities. Additionally, the Casimir energy in topological and Chern insulators has $d^{-3}$ 

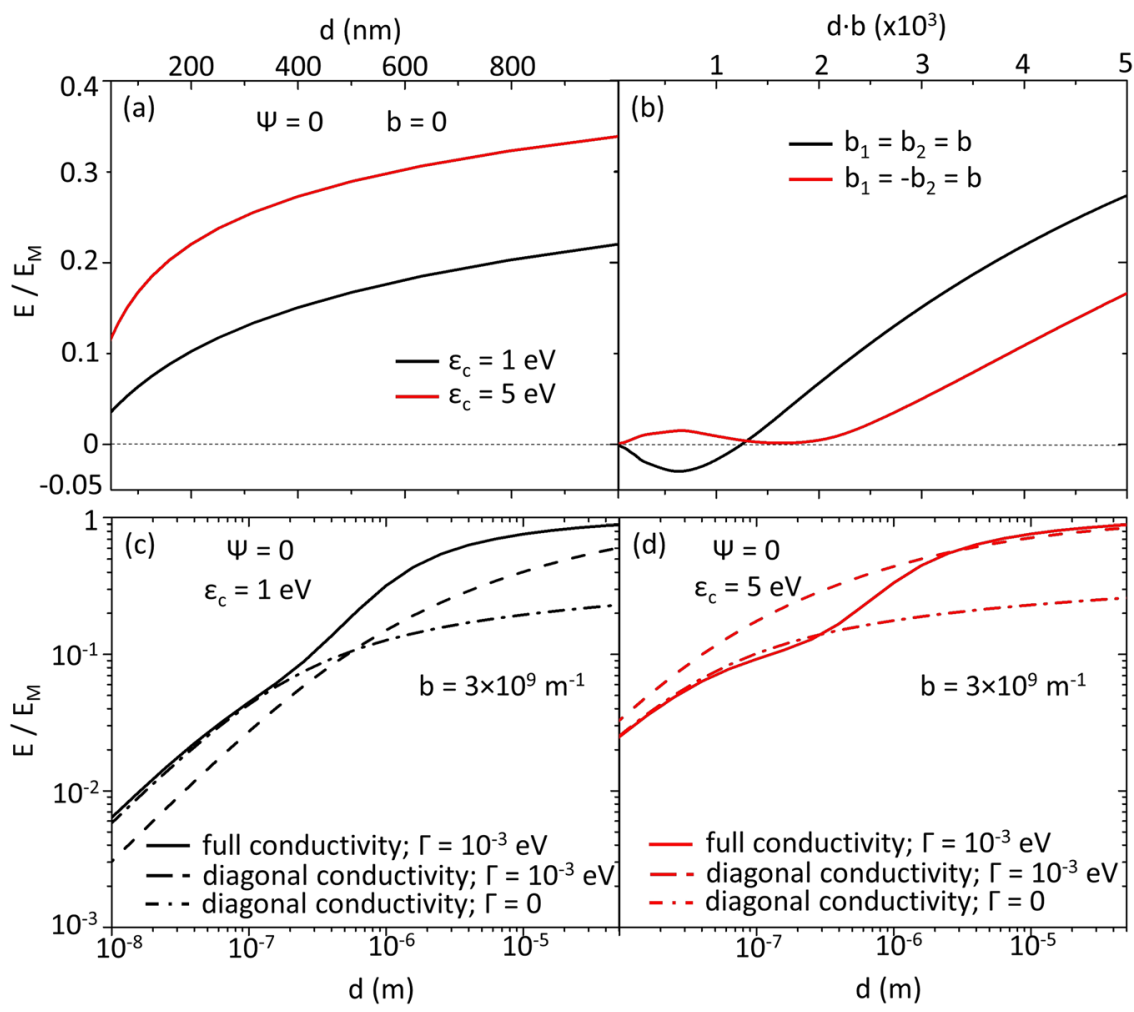

Fig. 4 Quantum mechanical Casimir energy for materials with $\boldsymbol{\Psi}=\mathbf{0}$ cones. The Casimir energy scaled by $E_{\mathrm{M}}=-\frac{\pi^{2} \hbar c}{720 d^{3}}$ as a function of: a distance for coinciding nontilted Weyl cones $(b=0, \Psi=0)$ and different values of the cutoff parameter $\epsilon_{\mathrm{c}} ; \mathbf{b}$ the unitless parameter $d \cdot b$ with bulk $\epsilon_{\mathrm{xx}}=\epsilon_{\mathrm{yy}}=\epsilon_{\mathrm{zz}}=1$ and no surface conductivity; $\mathbf{c}$, d distance for separated nontilted Weyl cones with $b_{1}=b_{2}=b$ calculated with the full conductivity (diagonal and Hall components) with $\Gamma=10^{-3} \mathrm{eV}$ (full curves), diagonal conductivity and no Hall components with $\Gamma=10^{-3} \mathrm{eV}$ (dashed curves), and diagonal conductivity and no Hall components with $\Gamma=0$ (dotted curves) for $\epsilon_{\mathrm{c}}=1 \mathrm{eV}$ and $\epsilon_{\mathrm{c}}=5 \mathrm{eV}$ respectively. Also, $b_{1,2}$ denote the cone separations of the two interacting Weyl materials.

scaling law, while in Weyl semimetals there is a crossover in distance dependence from $d^{-1}$ at small separations to $d^{-3}$ at large separations.

The numerical results for the interaction energy when only the bulk Hall conductivity $\sigma_{\mathrm{xy}, \mathrm{b}}^{\eta}=\sigma_{\mathrm{T}}=\frac{e^{2} b}{4 \pi^{2}}$ is taken into account are shown in Fig. $4 \mathrm{~b}$. The repulsive interaction at small separations is dictated by the sign of the $b_{1} b_{2}$ product according to Eq. (15), while at large separations the energy approaches the limit of perfect metals, which is in agreement with the above discussed analytical expressions. The strongest repulsion is found for $b \cdot d$ $\left(\times 10^{3}\right) \sim 1 / 2$, thus characteristic values $b=5-20 \mathrm{~nm}^{-1}$ correspond to $d=0.1-0.025$ microns. Nevertheless, this repulsive interaction is at least two orders of magnitude smaller than $E_{M}$ in the sub-micron and micron separation region (depending on $b$ values), as suggested by Fig. $4 \mathrm{~b}$.

In Fig. 4c, d the numerical results for the normalized Casimir energy as a function of separation calculated by taking into account the full optical conductivity tensor of dissipative materials are shown for two values of the cutoff energy. The corresponding energies obtained when only the diagonal components of the conductivity with and without dissipation are taken into account, are also shown. These results reveal the surprising role of dissipation: while the low and high $d$ limits in $E$ are best captured for calculations with the diagonal conductivity (no Hall components), the low $d$ limit is better described when $\Gamma=0$, while the higher $d$ range is better described when dissipation is finite. Figure $4 \mathrm{c}, \mathrm{d}$ further shows that the role of cone separation is to bring $E$ closer to $E_{\mathrm{M}}$ in the intermediate distance range and and all repulsive effects are washed out by the dominant diagonal components of the optical response.
At this point, one might ask how the surface conductivity due to the Fermi arcs affects the Weyl Casimir interaction. For the chosen $b_{\mu}=\left(0, b \hat{k}_{\mathrm{z}}\right) \sigma_{\mathrm{zz}, \mathrm{s}}=0$ as discussed earlier, thus to probe this property WSMs separated along the $y$-axis or $x$-axis must be considered with the appropriate Fresnel matrices given in the Supplementary Material. Although the numerical calculations are technically difficult we find that the surface conductivity plays even a lesser effect in the Casimir energy as compared to the one of the Hall conductivity as discussed above. The surface conductivity increases the metallic-like nature of the interaction, but given the smallness of the effect further results are not given.

Casimir interaction and cone tilting. In what follows, we further investigate how other characteristics affect the interaction between Weyl materials. More specifically, by utilizing the full optical response dissipative type I and type II WSMs separated along the $z$-axis are considered and the Casimir energy upon cutoff energy, degree of tilting, and chemical potential is calculated. For this purpose, the generalized Lifshitz formula in Eq. (20), while the optical response and Fresnel matrices can be found in the Supplementary Material. These results help us find ways to modulate the Weyl Casimir interaction as well as recognize signatures of the optical response and compare with the above discussed analytical expressions.

The scaling dependence shown in Fig. 5a indicates that Casimir energy increases as $d$ is increased, although for the case of $\Psi=0$, $\mu=0$ this increase is at a much smaller scale as compared to the other displayed cases. This behavior is reminiscent of the interaction between Drude-like metals controlled by the 


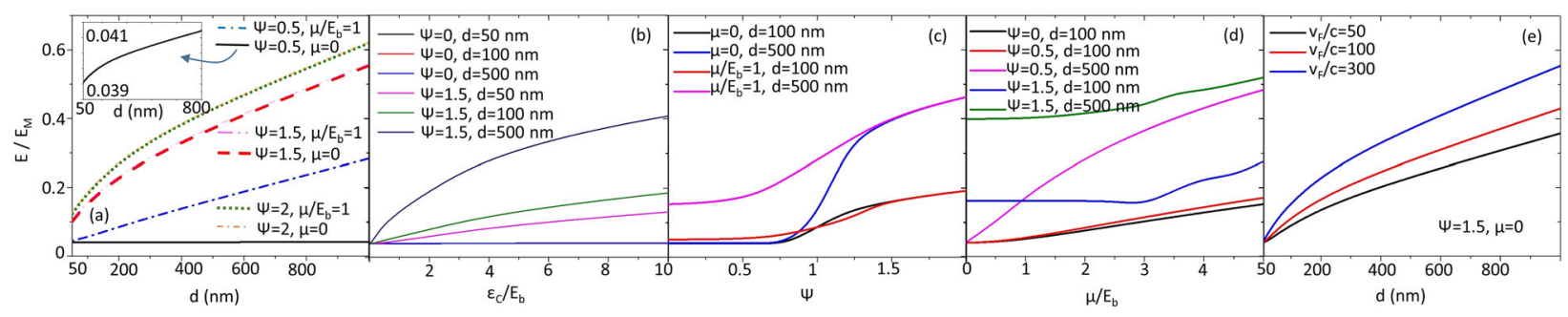

Fig. 5 Quantum Casimir energy for Weyl semimetals. The Casimir energy scaled by $E_{\mathrm{M}}=-\frac{\pi^{2} \hbar c}{720 d^{3}}$ for two identical Weyl semimetals separated along the $\mathrm{y}$-direction as a function of: a distance for different values of cone tilting and chemical potential $\left(\epsilon_{\mathrm{c}}=5 \mathrm{eV}, E_{\mathrm{b}}=1 \mathrm{eV}, \mathrm{c} / v_{\mathrm{F}}=300\right)$. The vertical axis of the inset is in log scale for better visibility; $\mathbf{b}$ cutoff energy scaled by $E_{\mathrm{b}}$ for different separations $\left(\epsilon_{\mathrm{c}}=5 \mathrm{eV}, \Psi=0, c / v_{\mathrm{F}}=300, \mu=0\right)$; (c) tilting for different separations and chemical potentials $\left(\epsilon_{\mathrm{c}}=5 \mathrm{eV}, E_{\mathrm{b}}=1 \mathrm{eV}, \mathrm{c} / v_{\mathrm{F}}=300, \mu=0\right)$; $\mathbf{d}$ chemical potential for different titling and separations $\left(\epsilon_{\mathrm{c}}=5\right.$ eV, $E_{\mathrm{b}}=$ $\left.1 \mathrm{eV}, \mathrm{c} / v_{\mathrm{F}}=300\right)$; e distance for different values of the Fermi velocity $\left(\epsilon_{\mathrm{c}}=5 \mathrm{eV}, E_{\mathrm{b}}=1 \mathrm{eV}, \mu=0, \Psi=0.5\right)$. In all calculations $\Gamma / E_{\mathrm{b}}=10^{-3}$.

magnitude of their plasma frequencies. The larger the plasma frequencies, the closer the interaction is to the one for perfect metals. In the case of larger $\Psi$ and $\mu$, the intraband conductivities dominate the Casimir interaction due to their large $\omega_{\mathrm{P}}$, while for $\Psi=0$ and $\mu=0$ the optical response is dominated by the interband components and $E / E_{\mathrm{M}}$ is a small fraction.

The behavior of $E / E_{\mathrm{M}}$ vs. $d$ for $\Psi=0, \mu=0$ in Fig. 5a is actually very similar to the analytical results of the Casimir energy for coinciding Weyl cones whose $d_{0}=20 \mathrm{~nm}$ when $\epsilon_{\mathrm{c}} / E_{\mathrm{b}}=5$ (Fig. 4a). Indeed, $E / E_{\mathrm{M}}$ at large distances $\left(d \gg d_{0}\right)$ is practically determined by the prefactor in Eq. (13), which indicates that type I WSM with $\mu=0$ behaves as a Dirac material with coinciding cones in the displayed region of distance separation. For the other cases in Fig. 5a, however, the nonzero plasma frequency elevates the role of the intraband conductivity and the interaction is essentially similar to the one of Drude-like metals ${ }^{2}$. Given that the cutoff energy plays a prominent role in $\omega_{\mathrm{P}}^{\mathrm{II}}$ Eqs. $(6,7)$, Fig. $5 \mathrm{~b}$ shows how the Casimir interaction changes as a function of $\epsilon_{\mathrm{c}}$ for type I and II WSMs. The much more pronounced enhancement of $E$ for type II WSMs as $\epsilon_{\mathrm{c}}$ is increased is due to the large intraband optical conductivity and specifically the strong dependence of its plasma frequency on the energy cutoff (Eqs. $(6,7))$. Figure $5 c$ displays how the Casimir energy changes as a function of degree of cone tilting. The change of an almost flat $E / E_{\mathrm{M}}$ to a linear-like behavior at $\Psi \sim 1$ signals a transition in the Casimir energy from type I to type II WSM. Again, the much stronger interaction for type II WSMs as compared to type I WSMs in Fig. 5c gives another perspective of the much enhanced role of the intraband optical conductivity for $\Psi>1$. Figure $5 \mathrm{~d}$ further shows that the chemical potential is also an effective parameter in controlling the magnitude of the Casimir energy for both types of WSMs, although the degree of tilting and distance separation can affect the degree of change. Additionally, increasing the Fermi velocity of the linear bands in Eq. (4) can increase the magnitude of Casimir energy although the characteristic behavior in terms of distance and other parameters are preserved (Fig. 5e).

Our numerical calculations indicate that the $b$ parameter plays a rather small role and the Casimir interaction is dominated by the contributions from the diagonal components in the optical tensor. This can be easily understood in the context of the above discussions for the limiting cases of coinciding Weyl cones and materials with only Hall conductivity components. In fact, taking the case of non-tilted Weyl cones and expanding the Fresnel reflection matrices for small $\sigma_{\mathrm{xy}}$ due to $\sigma_{\mathrm{xy}} \ll \sigma_{\mathrm{xx}}=\sigma_{\mathrm{zz}}$, we find that the first order correction (which contains $b$ ) in the small distance limit between Weyl semimetals with $\mu=0$ is the same as Eq. (15). Given the much longer range due to the $d^{-1}$ dependence and the $\alpha^{2}$ in the numerator, it becomes clear that first order correction is much smaller than the dominant Casimir energy due to the diagonal components of the bulk conductivity response.

Thermal Casimir energy. Thermal fluctuations in the Casimir energy are captured in the $n=0$ Matsubara term of Eq. (20) and they are expected to dominate at room temperature at separations on the sub-micron and micron scales for many materials ${ }^{2,4}$. To study the thermal effects in the Casimir interaction of WSMs, we calculate the conductivity at finite $T$ in the limit $\xi \rightarrow 0^{+}$using the results in the Supplementary Note 2 and the Maldague's formula $13,45,46$. The interband conductivity is linear in $\xi$ in the limit of small frequency and it is well approximated as

$$
\sigma_{\mathrm{ij}}(\mathrm{i} \xi) \approx \frac{2 \alpha c \xi}{3 \pi v_{\mathrm{F}}} \log \left(\frac{\epsilon_{c}}{\operatorname{Max}\left(|\mu|, \frac{\pi k_{\mathrm{B}} T}{2 e^{\gamma}}\right)}\right) \delta_{i j},
$$

where $\gamma$ is the Euler's constant. The intraband conductivity at finite $T$ and $\xi \rightarrow 0$ has the typical Drude-like expression

$$
\sigma_{\mathrm{ij}}(\mathrm{i} \xi) \approx \frac{\mathrm{c}^{2} \omega_{P}^{(\mathrm{I}, \mathrm{II}) 2}}{4 \pi(\xi+\Gamma)} \delta_{\mathrm{ij}},
$$

where $\omega_{\mathrm{P}}^{\mathrm{I}, \mathrm{II}}$ are the plasma frequencies for type I and type II WSMs. Since the optical response at $\xi \rightarrow 0^{+}$is dominated by the intraband contribution, the thermal Casimir energy is found as

$$
\frac{E_{\mathrm{T}}}{A}=-\frac{k_{\mathrm{B}} T}{16 \pi d^{2}} \zeta(3),
$$

where $\zeta(s)$ is the Riemann zeta function. Thus the thermal effects in the WSM Casimir interaction are of the usual form of a Drude metal.

Experimental measurements of the Casimir interaction in Weyl semimetals can be realized using a setup based on an atomic force microscope, which has been used for several other materials such as noble metals, ferromagnets, oxides, and graphene covered substrates ${ }^{12,47,48}$. Such a setup allows measuring a Casimir force whose magnitude is a fraction of the one of perfect metals and probe separations in the submicron-micron range. By taking type I and II Weyl semimetals one can potentially measure the interaction as a function of distance and get information on the role of cone tilting. By doping the materials or applying external gate voltage, one can also probe the role of the chemical potential. For distance separations in the submicron-micron range the linear band approximation dominates the Casimir interaction in most Weyl semimetals which ensures valid comparison with our theory. Due to the similarity of Weyl materials with metallic systems it is expected that thermal effects will not be important at such distances and the atomic force microscope technology at room temperature ${ }^{12,47,48}$ will capture the quantum mechanical limit of the interaction. 
In this study, we present a comprehensive investigation of the bulk and surface conductivity components by distinguishing between type I and type II WSMs, which are then applied to the quantum mecahnical and thermal limits of the Casimir interaction. Our results indicate that although there is a complex interplay between the electronic and optical properties of these materials, the interaction exhibits a behaviors similar to the one of metallic-like systems due to the dominant role of the diagonal bulk conductivity components. The quantum mechanical energy can be modulated as a function of chemical potential and cone tilting as captured by the $\mu$ and $\Psi$ dependences in the plasma frequency for type I and type II materials. The explicit $\epsilon_{\mathrm{c}}$ dependence in the bulk conductivity renders strong dependence of the energy upon the cutoff corresponding to the validity of the linear energy dispersion. For separations larger than the characteristic distance $d_{0}$ set by $\epsilon_{\mathrm{c}}$ the linear energy dispersion will dominate the interaction, while for larger than $d_{0}$ separations other energy bands are expected to be important and have to be considered in the electronic and optical properties of the materials. Unlike topological and Chern insulator, where the nontrivial topology can result in significant repulsive and even quantization effects, the anomalous Hall conductivity plays a rather small role in the quantum interaction in Weyl materials. We also find that because of the dominant diagonal bulk conductivity, thermal fluctuations are expected to affect the Casimir interaction in a similar way as the case of metallic systems. Our investigation is a testament that a thorough understanding of the fundamental electronic and optical properties of materials is necessary in order to make progress towards other research areas, such as light-matter interactions and the Casimir force as a particular example.

\section{Methods}

Kubo formalism. The optical response of a given material is directly related to its electronic structure. The dynamical optical conductivity tensor is a fundamental property, which is a necessary ingredient in understanding light-matter interactions, such as the Casimir force. The components of this tensor can be calculated using the Kubo formalism ${ }^{49}$. For a given cone specified by its chirality $\eta$, these are

$$
\sigma_{\mathrm{ij}}^{\eta}(\omega, \mathbf{q})=-i e^{2} \sum_{\lambda, \lambda^{\prime}} \int \frac{d^{d} \mathbf{k}}{(2 \pi)^{d}} \frac{<u_{\mathbf{k}}^{\lambda, \eta}\left|v_{i}^{\eta}\right| u_{\mathbf{k}+\mathbf{q}}^{\lambda^{\prime}, \eta}><u_{\mathbf{k}+\mathbf{q}}^{\lambda^{\prime}, \eta}\left|v_{j}^{\eta}\right| u_{\mathbf{k}}^{\lambda, \eta}>n_{\mathrm{F}}\left(E_{\mathbf{k}}^{\lambda, \eta}\right)-n_{\mathrm{F}}\left(E_{\mathbf{k}+\mathbf{q}}^{\lambda^{\prime}, \eta}\right)}{\hbar(\omega+i / \tau)+E_{\mathbf{k}}^{\lambda, \eta}-E_{\mathbf{k}+\mathbf{q}}^{\lambda^{\prime}, \eta}} .
$$

Here $\mid u_{\mathbf{k}}^{\lambda, \eta}>$ and $E_{\mathbf{k}}^{\lambda, \eta}\left(\lambda, \lambda^{\prime}= \pm\right.$ correspond to the electron and hole states $)$ are the eigenspinors and eigenenergies for the Hamiltonian in Eqs. (3-5) and $\mathbf{v}^{\eta}=$ $\nabla_{k} H_{s}^{\eta} / \hbar=\eta v_{\mathrm{F}}\left(\sigma_{\mathrm{x}}, \sigma_{\mathrm{y}}, \sigma_{\mathrm{z}}\right)$ is the velocity operator. Also, the Fermi-Dirac distribution function is $n_{\mathrm{F}}\left(E_{\mathbf{k}}^{\lambda, \eta}\right)=1 /\left(e^{\left(E_{k}^{\lambda}-\mu\right) / k_{\mathrm{B}} T}+1\right)$ and $\tau$ is the relaxation time.

Lifshitz formalism. The Casimir interaction can be calculated using the Lifshitz approach $^{2,4}$, however for lossy materials with reflection matrices with complex frequency dependence this formalism must be applied carefully. Specifically, one must account for contributions from propagating and evanescent waves, which can arise from frequencies above and below the light cone, respectively ${ }^{50}$. Thus, for semi-infinite planar objects taken to be separated by a distance $d$ along the $z$-axis, the interaction energy per unit area $A$ can be written as

$$
\begin{gathered}
\frac{E}{A}=\frac{\mathcal{E}}{A}+\frac{\mathcal{E}^{*}}{A}, \\
\frac{\mathcal{E}}{A}=k_{\mathrm{B}} T \sum_{n}^{\prime} \int \frac{d^{2} \mathbf{k}_{\|}}{(2 \pi)^{2}} \log \operatorname{det}\left(1-\mathbf{R}_{1} \cdot \mathbf{R}_{2}^{\prime} \mathrm{e}^{-2 \mathrm{k}_{\mathrm{n}} \mathrm{d}}\right) .
\end{gathered}
$$

Here $\mathbf{R}_{j}=\mathbf{R}_{j}\left(k_{\mathrm{n}}, k_{\mathrm{x}}, k_{\mathrm{y}}\right)$ for $j=1,2$ are the $2 \times 2$ Fresnel reflection matrices for the interacting objects, where $k_{n}=\sqrt{k_{\mathrm{x}}^{2}+k_{\mathrm{y}}^{2}+\xi_{n}^{2} / c^{2}}$ with $\xi_{n}=2 \pi n k_{\mathrm{B}} T / \hbar$ being the Matsubara frequencies $\left(n=0,1,2, \ldots\right.$ and $\left.\mathbf{k}_{\|}=\left(k_{\mathrm{x}}, k_{\mathrm{y}}\right)\right)$. Also, $\mathbf{R}_{j}^{\prime}=\mathbf{R}_{j}\left(-k_{\mathrm{n}}, k_{\mathrm{x}}, k_{\mathrm{y}}\right)$ denotes the reflection matrix for scattering with opposite direction ${ }^{17}$. The generalized Lifshitz expression from Eqs. (20) is necessary for the interaction involving Weyl semimetals. From our subsequent calculations we find that the reflection matrices may not be real in some imaginary frequency ranges. This is attributed to the interplay between propagating and evanescent wave stemming from the anisotropic optical response, which motivates the generalization of the Lifshitz formalism ${ }^{50}$.

The reflection matrices depend explicitly on the optical response of the involved materials and they are obtained by applying electromagnetic boundary conditions for semi-infinite 3D objects with planar surfaces separated at a finite distance. Resolving the reflection matrices is technically difficult as each type of Weyl semimetal has anisotropic bulk and surface optical response as well as nonzero bulk Hall response. Nevertheless, the electric and magnetic fields can be expanded in terms of the suitable for planar geometry orthogonal functions $\boldsymbol{M}_{\boldsymbol{k}}(\boldsymbol{r})=$

$\frac{1}{k_{\|}} \nabla \times\left[\hat{\boldsymbol{z}} \phi_{\boldsymbol{k}}(\boldsymbol{r})\right]$ and $\boldsymbol{N}_{\boldsymbol{k}}(\boldsymbol{r})=\frac{c}{\omega} \nabla \times \boldsymbol{M}_{\boldsymbol{k}}(\boldsymbol{r})\left(\phi_{\boldsymbol{k}}(\boldsymbol{r})=e^{\mathrm{i} \boldsymbol{k} \cdot \boldsymbol{r}}, k_{\|}=\sqrt{k_{\mathrm{x}}^{2}+k_{\mathrm{y}}^{2}}\right)$. Details of the derivation of the electromagnetic radiation impinging on the $x=0, y=0$, or $z=0$ surface of a semi-infinite Weyl material can be found in Supplementary Notes 3, 4 .

\section{Data availability}

The data that support the plots in the paper and other findings of this study are available from the corresponding author upon reasonable request.

Received: 4 November 2019; Accepted: 26 February 2020; Published online: 26 March 2020

\section{References}

1. Casimir, H. B. G. On the attraction between two perfectly conducting plates. Proc. K. Ned. Akad. Wet. 51, 793 (1948).

2. Klimchitskaya, G. L., Mohideen, U. \& Mostepanenko, V. M. The Casimir force between real materials: experiment and theory. Rev. Mod. Phys. 81, 1827 (2009)

3. Chan, H. B., Aksyuk, V. A., Kleiman, R. N., Bishop, D. J. \& Capasso, F. Quantum mechanical actuation of microelectromechanical systems by the Casimir force. Science 291, 1941 (2001).

4. Woods, L. M. et al. Materials perspective on Casimir and van der Waals interactions. Rev. Mod. Phys. 88, 045003 (2016).

5. Bordag, M., Geyer, B., Klimchitskaya, G. L. \& Mostepanenko, V. M. Lifshitz type formulas for graphene and single-wall carbon nanotubes: van der Waals and Casimir interactions. Phys. Rev. B 74, 205431 (2006).

6. Gomez-Santos, G. Thermal van der Waals interaction between graphene layers. Phys. Rev. B 80, 245424 (2009).

7. Drosdoff, D. \& Woods, L. M. Casimir forces and graphene sheets. Phys. Rev. B 82, 155459 (2010).

8. Sarabadani, J., Naji, A., Asgari, R. \& Podgornik, R. Many-body effects in the van der Waals-Casimir interaction between graphene layers. Phys. Rev. B 84, 155407 (2011)

9. Svetovoy, V., Moktadir, Z., Elwenspoek, M. \& Mizuta, H. Tailoring the thermal Casimir force with graphene. EPL 96, 14006 (2011).

10. Khusnutdinov, N., Kashparov, R. \& Woods, L. M. Thermal Casimir and Casimir-Polder interactions in $\mathrm{N}$ parallel 2D dirac materials. 2D Mater 5, 035032 (2018).

11. Fialkovsky, I. V., Marachevsky, V. N. \& Vassilevich, D. V. Finite temperature Casimir effect for graphene. Phys. Rev. B 84, 035446 (2011).

12. Banishev, A. A., Klimchitskaya, G. L., Mostepanenko, V. M. \& Mohideen, U. Demonstration of the Casimir force between ferromagnetic surfaces of a Nicoated sphere and a Ni-coated plate. Phys. Rev. Lett. 110, 137401 (2013).

13. Rodriguez-Lopez, P., Kort-Kamp, W. J. M., Dalvit, D. A. R. \& Woods, L. M. Casimir force phase transitions in the graphene family. Nat. Commun. 8, 14699 (2017).

14. Grushin, A. G. \& Cortijo, A. Tunable Casimir repulsion with threedimensional topological insulators. Phys. Rev. Lett. 106, 020403 (2011).

15. Rodriguez-Lopez, P. \& Grushin, A. G. Repulsive Casimir effect with Chern insulators. Phys. Rev. Lett. 112, 056804 (2014).

16. Wilson, J. H., Allocca, A. A. \& Galitski, V. Repulsive Casimir force between Weyl semimetals. Phys. Rev. B 91, 235115 (2015).

17. Fialkovsky, I. V., Khusnutdinov, N. \& Vassilevich, D. Quest for Casimir repulsion between Chern-Simons surfaces. Phys. Rev. B 97, 165432 (2018)

18. Hasan, M. Z., Xu, S.-Y., Belopolsky, I. \& Huang, S.-M. Discovery of Weyl fermion semimetals and topological fermi arc states. Ann. Rev. Cond. Matter Phys. 8, 289 (2017).

19. Wan, X., Turner, A. M., Vishwanath, A. \& Savrasov, S. Y. Topological semimetal and Fermi-arc surface states in the electronic structure of pyrochlore iridates. Phys. Rev. B 83, 205101 (2011).

20. Gorbar, E. V., Miransky, V. A., Shovkovy, I. A. \& Sukhachov, P. O. Origin of dissipative Fermi arc transport in Weyl semimetals. Phys. Rev. B 93, 235127 (2016).

21. Armitage, N. P., Mele, E. J. \& Vishwanath, A. Weyl and Dirac semimetals in three-dimensional solids. Rev. Mod. Phys. 90, 015001 (2018).

22. Yan, B. \& Felser, C. Topological materials: Weyl semimetals. Annu. Rev. Cond. Matter Phys. 8, 337 (2016). 
23. Osterhoudt, G. B. et al. Colossal mid-infrared bulk photovoltaic effect in a type-I Weyl semimetal. Nat. Mater. 18, 471 (2019).

24. Soluyanov, A. A. et al. Type-II Weyl semimetals. Nature 527, 495 (2015).

25. Yu, Z.-M., Yao, Y. \& Yang, S. A. Predicted unusual magnetoresponse in typeII Weyl semimetals. Phys. Rev. Lett. 117, 077202 (2016).

26. Zyuzin, A. A. \& Tiwari, R. P. Intrinsic anomalous Hall effect in type-II Weyl semimetals. JETP Lett. 103, 717 (2012).

27. Carbotte, J. P. Dirac cone tilt on interband optical background of type-I and type-II Weyl semimetals. Phys. Rev. B 94, 165111 (2016)

28. Mukherjee, S. P. \& Carbotte, J. P. Imaginary part of Hall conductivity in a tilted doped Weyl semimetal with both broken time-reversal and inversion symmetry. Phys. Rev. B 97, 035144 (2018).

29. Tabert, C. J. \& Carbotte, J. P. Optical conductivity of Weyl semimetals and signatures of the gapped semimetal phase transition. Phys. Rev. B 93, 085442 (2016).

30. Carbotte, J. P. Dirac cone tilt on interband optical background of type-I and type-II Weyl semimetals. Phys. Rev. B 94, 165111 (2016).

31. Mukherjee, S. P. \& Carbotte, J. P. Imaginary part of Hall conductivity in a tilted doped Weyl semimetal with both broken time-reversal and inversion symmetry. Phys. Rev. B 97, 035144 (2018).

32. Mukherjee, S. P. \& Carbotte, J. P. Absorption of circular polarized light in tilted type-I and type-II Weyl semimetals. Phys. Rev. B 96, 085114 (2017).

33. Zyuzin, A. A. \& Tiwari, R. P. Intrinsic anomalous Hall effect in type-II Wyl semimetals. JETP Lett. 103, 717 (2016).

34. Rosenstein, B. \& Lewkowicz, M. Dynamics of electric transport in interacting Weyl semimetals. Phys. Rev. B 88, 045108 (2013).

35. Kotov, O. V. \& Lozovik, Y. E. Dielectric response and novel electromagnetic modes in three-dimensional Dirac semimetal films. Phys. Rev. B 93, 235417 (2016).

36. Rodriguez-Lopez, P., Kort-Kamp, W. J. M., Dalvit, D. A. R. \& Woods, L. M. Nonlocal optical response in topological phase transitions in the graphene family. Phys. Rev. Mater. 2, 014003 (2018).

37. Inglesfield, J. E. Surface electronic structure. Rep. Prog. Phys. 45, 223 (1982).

38. Wang, Z., Weng, H., Wu, Q., Dai, X. \& Fang, Z. Three-dimensional Dirac semimetal and quantum transport in $\mathrm{Cd}_{3} \mathrm{As}_{2}$. Phys. Rev. B 88, 125427 (2013).

39. $\mathrm{Xu}, \mathrm{S}$.-Y. et al. Discovery of a Weyl fermion semimetal and topological Fermi arcs. Science 349, 613 (2015).

40. McCormick, T. M., Kimchi, I. \& Trivedi, N. Minimal models for topological Weyl semimetals. Phys. Rev. B 95, 075133 (2017).

41. Mathai, V. \& Thiang, G. C. Global topology of Weyl semimetals and Fermi arcs. J. Phys. A 50, 11LT01 (2017).

42. Mong, R. S. K. \& Shivamoggi, V. Edge states and the bulk-boundary correspondence in Dirac Hamiltonians. Phys. Rev. B 83, 125109 (2011).

43. Buividovich, P. V. Surface states of massive Dirac fermions with separated Weyl nodes. https://arxiv.org/abs/1411.7543v2 (2015).

44. Grushin, A. G., Venderbos, J. W. F. \& Bardarson, J. H. Coexistence of Fermi arcs with two-dimensional gapless Dirac states. Phys. Rev. B 91, 121109 (2015).

45. Giuliane, G. F. \& Vignale, G., Quantum Theory of the Electron Liquid. (Cambridge University Press, 2005).

46. Maldague, P. F. Many-body corrections to the polarizability of the twodimensional electron gas. Surf. Sci. 73, 296 (1978).

47. Decca, R. S. et al. Tests of new physics from precise measurements of the Casimir pressure between two gold-coated plates. Phys. Rev. D 75, 077101 (2007).
48. Banishev, A. A. et al. Measuring the Casimir force gradient from graphene on a $\mathrm{SiO}_{2}$ substrate. Phys. Rev. B 87, 205433 (2013).

49. Mahan, G. D. Many-Particle Physics. (Kluwer Academic/Plenum, New York, 2000).

50. Genet, C., Lambrecht, A. \& Reynaud, S. Casimir force and the quantum theory of lossy optical cavities. Phys. Rev. A 67, 043811 (2003).

\section{Acknowledgements}

P.R.-L. acknowledges partial support from TerMic (Grant No. FIS2014-52486-R, Spanish Government), CONTRACT (Grant No. FIS2017-83709-R, Spanish Government, from Juan de la Cierva-Incorporacion program (Ref: I JCI-2015-25315, Spanish Government), MAT2015-66888-C3-1R and RTI2018-097895-B-C41 funded by the Spanish Government (Ministerio de Ciencia, Innovación y Universidades) and by the Feder Program of the European Union. N.K. was supported in parts by the grants 2016/033196, 2017/50294-1 and 2019/10719-9 of the São Paulo Research Foundation (FAPESP), by the RFBR projects 19-02-00496-a. L.M.W. acknowledges funcial support from the US Department of Energy under grant No. DE-FG02-06ER46297. Helpful discussions with Drs. Pablo San-Jose, Alberto Cortijo and Adolfo G. Grushin are also acknowledged.

\section{Author contributions}

P.R.-L., A.P., I.F., N.K., and L.M.W. contributed equally to this work.

\section{Competing interests}

The authors declare no competing interests.

\section{Additional information}

Supplementary information is available for this paper at https://doi.org/10.1038/s43246020-0015-4.

Correspondence and requests for materials should be addressed to L.M.W.

Reprints and permission information is available at http://www.nature.com/reprints

Publisher's note Springer Nature remains neutral with regard to jurisdictional claims in published maps and institutional affiliations.

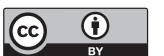

Open Access This article is licensed under a Creative Commons Attribution 4.0 International License, which permits use, sharing, adaptation, distribution and reproduction in any medium or format, as long as you give appropriate credit to the original author(s) and the source, provide a link to the Creative Commons license, and indicate if changes were made. The images or other third party material in this article are included in the article's Creative Commons license, unless indicated otherwise in a credit line to the material. If material is not included in the article's Creative Commons license and your intended use is not permitted by statutory regulation or exceeds the permitted use, you will need to obtain permission directly from the copyright holder. To view a copy of this license, visit http://creativecommons.org/ licenses/by/4.0/.

(C) The Author(s) 2020 